\title{
Ventral Mesencephalon-Enriched Genes That Regulate the Development of Dopaminergic Neurons In Vivo
}

\author{
Min Yin, ${ }^{1,2 \star}$ Shuxi Liu, ${ }^{2 \star}$ Yanqing Yin, ${ }^{2}$ Sen Li, ${ }^{2}$ Zhihua Li, ${ }^{1}$ Xuefei Wu, ${ }^{1}$ Bo Zhang, ${ }^{2}$ Siew-Lan Ang, ${ }^{3}$ Yuqiang Ding, ${ }^{2}$ and \\ Jiawei Zhou ${ }^{2}$ \\ ${ }^{1}$ Laboratory of Molecular Cell Biology, Institute of Biochemistry and Cell Biology, and ${ }^{2}$ State Key Laboratory of Neuroscience, Shanghai Institutes for \\ Biological Sciences, Chinese Academy of Sciences, Shanghai 200031, China, and ${ }^{3}$ Division of Developmental Neurobiology, Medical Research Council \\ National Institute for Medical Research, London NW7 1AA, United Kingdom
}

\begin{abstract}
Mesodiencephalic dopaminergic (mDA) neurons are critical for movement control and other physiological activities. However, the molecular mechanisms underlying their development are poorly understood. We aimed to establish the expression profiles of genes involved in this process and unravel genetic programs that control late development of mDA neurons. We compared genome-wide gene expression profiles of developing mouse ventral mesencephalon (VM) using microarrays. We identified a set of genes that show spatially and temporally restricted expression in the VM in an $\mathrm{Ngn} 2$ (neurogenin 2)-dependent manner and are potentially important for mDA neuron development. Functional analysis on mice lacking the VM-specific gene early B-cell factor 1 (Ebf1) revealed that Ebf1 is essential for the terminal migration of mDA neurons in the substantia nigra pars compacta. Thus, we identified a set of VM-enriched genes that are important for mDA neuron development. Our analysis also provides a genetic framework for further investigation of the molecular mechanisms mediating mDA neuron development.
\end{abstract}

\section{Introduction}

Mesodiencephalic dopaminergic (mDA) neurons are essential for the control of multiple brain functions, including movement control, emotion, and reward. Dysfunction of the mDA system is involved in the pathogenesis of several mental and neurological diseases, such as Parkinson's disease (PD) and schizophrenia, of which some are considered to have a neurodevelopmental origin. Currently no effective treatment is available for PD that alters progression of the disease itself. Transplantation of human fetal mesencephalic tissue containing immature mDA neurons has demonstrated beneficial effects in PD patients (Björklund, 2005). However, this promising approach suffers from several drawbacks, for example, lack of sources of donor tissue, poor survival and migration of transplanted mDA neurons, and limited integration with host brain. Because of poor understanding of the molecular mechanisms controlling mDA neuron development,

Received Nov. 20, 2008; accepted Feb. 7, 2009.

This work was supported by grants from State Key Program for Basic Research of China (Nos. 2006CB500704 2007(B947100), "863" Research Program (No. 2006AA02A114), Natural Science Foundation of China (Nos. 30525041, 30623003, 30621130075), and Shanghai Metropolitan Fund for Research and Development (No. 07DJ14005). We thank Dr. R. Grosschedl (University of Texas Health Sciences (enter, Houston, TX) for providing Ebf1 null mice, Dr. F. Guillemot (Medical Research Council National Institute for Medical Research, London, UK) for Ngn2 null mice, and Dr. R. L. Johnson (University of Texas Health Sciences Center, Houston, TX) for $L m x 1 b$ null mice. We thank Dr. P. Smidt (University Medical Center Utrecht, Utrecht, The Netherlands) for Pitx3 pAb, Dr. M. German (University of California, San Francisco, San Francisco, CA) for Lmx1a pAb, and Dr. F. G. Rathjen (Max Delbruck Center for Molecular Medicine-Berlin-Buch, Berlin, Germany) for L1 pAb. We are also very grateful to Drs. L.P. Cheng, N. H. Jing, W. Bian, and X. Y. Wang (Institute of Biochemistry and Cell Biology, Shanghai, China) for their help with this project. We thank R. Z. Yang for drawing the diagram.

${ }^{*} M$.Y. and S.L. contributed equally to this work.

Correspondence should be addressed to Dr. Jiawei Zhou, Institute of Neuroscience, Chinese Academy of Sciences, 320 Yueyang Road, Shanghai 200031, China. E-mail: jwzhou@ion.ac.cn.

DOI:10.1523/JNEUROSCI.5569-08.2009

Copyright $\odot 2009$ Society for Neuroscience $\quad$ 0270-6474/09/295170-13\$15.00/0 attempts at restorative treatment of PD using neural transplantation and drug therapy have been hampered in the last two decades. Stem/neural progenitor cells have recently received much attention as they have the potential to generate large numbers of DA neurons for neural transplantation (McKay and Kittappa, 2008). However, the application of stem cell-derived mDA neurons in PD treatment likely faces similar problems. Thus, studies on mDA neuron development, including their specification, differentiation, migration, and survival, will promote our understanding of mechanisms of brain development and have an impact on developing novel approaches for the treatment of neurodegenerative diseases such as PD.

The development of mDA neurons occurs in the embryonic ventral mesencephalon (VM), which develops into two structurally separated nuclei in the adult mouse brain, the substantia nigra (SN) and ventral tegmental area (VTA). This highly dynamic process has been subdivided into three distinct phases. First is the induction of a progenitor field within the neuroepithelium that is competent to generate $\mathrm{mDA}$ precursors at early stages of neural development [approximately from embryonic day 8.5 (E8.5) to E10.5]. Neurogenin 2 (Ngn2), Lmx1a, Lmx1b, and Mash 1 are considered to be the early fate determinants of mDA neurons (Ang, 2006). Second is the specification and early differentiation of an mDA neuronal fate at intermediate stages (approximately from E10.5 to E12.5). Third is terminal differentiation of $\mathrm{mDA}$ neurons to acquire the mature phenotype, and outgrowth of axons to their targets at relatively late stages of neural development (after E12.5) (Prakash and Wurst, 2006). Nurr1 and Pitx3 are more concerned with the mDA neuron differentiation, survival, and DA neuron phenotype formation (Smidt and Burbach, 2007). Despite tremendous progress on the 
identification of transcription factors and fate determinants that control the processes of early mDA neuron development, the genetic program governing late $\mathrm{mDA}$ neuron development including differentiation, migration, and axonal pathfinding is largely unknown.

In the present study, we investigated the molecular events of mDA neuron development. The VM tissues analyzed were collected on five consecutive days (E10-E14): a critical window spanning key events of mDA cell specification, differentiation, migration, and connectivity. We identified a set of genes that is enriched in the VM. Genetic pathway analysis and in vivo functional studies in mutant mice demonstrated that these genes are novel factors controlling mDA development.

\section{Materials and Methods}

Tissue preparation and isolation of VM tissues. Mice were maintained according to guidelines of the Ethical Committee for animal use at the Shanghai Institutes for Biological Sciences. Timed matings were set up between adult mice of strain either ICR or C57BL/6. The morning of vaginal plug identification was designated E0. Embryos were collected in ice-cold HBSS, and ventral midbrains from embryos at E10, E11, E12, E13, and E14 were dissected using sharp needles as described previously (Simon et al., 2001). Tissues were immediately frozen in liquid nitrogen and stored at $-80^{\circ} \mathrm{C}$ until use. E10 and E11 VM tissue was collected from approximately 35 pregnant mice for each time point, and E12, E13, and E14 VMs were from an average of 15 pregnant mice for each time point.

Total RNA preparation and array hybridization. Total RNA was isolated from embryonic ventral midbrain tissue using Trizol (Invitrogen) and purified using RNeasy columns (Qiagen). Double-stranded cDNA was synthesized from total RNA, and the cDNA was used as a template for in vitro transcription to generate biotin-labeled cRNA using the High Yield RNA Transcript Labeling Kit (Qiagen). The labeled cRNA was purified by using RNeasy columns (Qiagen) and then fragmented. Affymetrix MOE430A and MOE430B array sets were used for all hybridizations (Affymetrix). MOE430A contained 22,626 probe sets analyzing $>14,000$ known genes and MOE430B contained 22,511 expressed sequence tag (EST) probe sets. Fifteen ventral midbrain samples from E10, E11, E12, E13, and E14 (triplicates) were hybridized to 30 chip sets (15 chips for MOE430A, 15 chips for MOE430B). The same samples were hybridized to both MOE430A and MOE430B at every time point. The array for every time point was repeated three times with different batches of sample. All RNA samples were subjected to RNA quality control by inspection on an agarose gel, measurement of 260/280 nm absorbance ration, and prehybridization using the Test Chips (Affymetrix) to ensure the highest-quality RNA. Array hybridization was performed following the manufacturer's protocol, and the arrays were scanned with a laser scanner (Affymetrix).

Data analysis. The CEL files were obtained using GCOS (Genechip operating software, Affymetrix) from DAT files. The mean correlation coefficient was calculated. Then the CEL files were analyzed in the $\mathrm{R}$ computing environment (2.1.1) using the linear models for the microarray data package (affylmGUI, 1.3.1), which is part of the Bioconductor project (www.Bioconductor.org) and is suitable for analyzing time-point microarray data. Under affylmGUI, "RMA" was selected as the normalization method and the data were analyzed using the computed linear model. Differentially expressed genes were ranked in decreasing order of the $B$ statistic. To identify the differential expression genes more accurately, the microarray data were also separately processed with the significance analysis of microarrays (SAM) (time course) method. The intersection of differentially expressed genes analyzed by these two statistical methods was selected for clustering. A total of 1285 differential expressed genes [linear models for microarray (limmar) $p=0.01$ and SAM $\Delta=$ 0.4 ] were chosen for hierarchical clustering.

In situ hybridization and immunohistochemistry. Embryos were fixed overnight in $4 \%$ paraformaldehyde at $4^{\circ} \mathrm{C}$. In situ hybridization (ISH) was performed on cryosections ( $12 \mu \mathrm{m}$ thick) with digoxigenin-labeled single-stranded RNA probes as described previously (Ma et al., 1998).
Probes for ISH were 300-400 bp long. Sequences of primers used in in situ hybridization are shown in supplemental Table S1 (available at www. jneurosci.org as supplemental material).

Procedures for immunohistochemistry and information on the primary antibodies are detailed in the supplemental methods (available at www.jneurosci.org as supplemental material).

Statistical analysis. Statistical analysis was performed using statistical software (GraphPad Prism v4.0, GraphPad Software). The control group was compared with the different treatment groups using ANOVA or unpaired Student's $t$ test. Differences were considered significant when $p$ values were $<0.05$.

\section{Results}

Microarray data processing and gene expression profiling of the developing VM

First, the microarray data were analyzed for reproducibility. The correlation coefficient for five consecutive time points (E10E14) in biological triplicates ranged from 0.96 to 0.99 with a mean correlation coefficient of 0.97 between three replicates, demonstrating a high degree of reproducibility. A series of genes was detected that are known to be critical for the specification and differentiation of mDA neurons as well as for DA biosynthesis and release, suggesting high reliability of the data (data not shown). This ensured a high likelihood of identifying differentially expressed genes involved in $\mathrm{mDA}$ neuron development from the microarray data.

To identify those differentially expressed genes reliably, two different but complementary statistical methodologies, limmar and SAM, were used to analyze the microarray data further. We assessed the significance of differences in gene expression by pairwise comparisons at each embryonic age. Those genes, whose levels were altered at least twofold in each pairwise comparison (10 comparisons in total for five time points), and intersected by the two statistical methodologies, were selected for further analysis. Up to 1285 such genes were selected (limmar $p=0.01$, SAM $\Delta=0.4)$. Among them, expression levels of 979 genes were upregulated, whereas 306 genes were downregulated during E10-E14.

Quantitative analysis showed that the most remarkable change in the number of gene transcripts (215) between two adjacent embryonic ages occurred between E11 and E12. Gene expression variation was much less common between E10 and E11 as well as between E13 and E14 (supplemental Fig. S1 A, B, available at www.jneurosci.org as supplemental material). No genes were detected whose levels were altered more than twofold between E12 and E13 or between E13 and E14. These data suggest that VM cells between E10 and E14 share common genetic determinants at different stages of development. E12 is a turning point for gene expression variation during VM development with greater fluctuation in gene expression during E10-E12 and less variation from E12-E14.

\section{VM cells of different embryonic age share clusters of developmentally regulated genes}

It has been shown that multiple genetic cascades control the development of $\mathrm{mDA}$ neurons in an $\mathrm{mDA}$ neuron-specific or -nonspecific manner. To obtain a global view of the first set of microarray data, we first performed hierarchical clustering analysis to reveal changes in gene expression while mDA neurons undergo differentiation. We found that the majority of genes were expressed in the VM at each embryonic age examined, but with different profiles, given that their levels were specifically upregulated or downregulated during development (supplemental Fig. S1 $B-D$, available at www.jneurosci.org as supplemental 
material). This suggests that $\mathrm{mDA}$ neuron development is regulated by a developmental-specific genetic network. In this context, combinations of a small set of genes, whose expression is embryonic age dependent, may play critical roles at a specific developmental stage. Together with the data shown in supplemental Figure S1A (available at www.jneurosci.org as supplemental material), this analysis also shows that the VMs at each embryonic age were molecularly similar to each other. In particular, gene expression profiles between adjacent ages (E10 vs E11, E13 vs E14) were extremely highly similar. This further emphasizes the significance of a small set of differentially expressed genes in VM development.

\section{Identification of VM-enriched genes}

To identify VM-enriched genes, 162 upregulated and 24 downregulated genes were selected for further analysis from 1285 differentially expressed genes identified in microarray data. Their trends of expression were analyzed individually. As a result, a set of VM-enriched genes was identified, and expression trends of some of the potentially important genes are shown in Figure 1. These genes fall into one of three categories based on expression profiles, which is suggestive of a specific role in a distinct aspects of VM development: (1) genes whose levels were significantly upregulated or downregulated in early development of $\mathrm{mDA}$ neuron (E10-E11) (Fig. 1A), and might be important for the maintenance of DA precursor cells status; (2) genes whose levels were significantly altered in intermediate development of $\mathrm{mDA}$ neurons (E11-E12) (Fig. 1B) and might be required for withdrawal of mDA precursor cells from mitosis and initiation of postmitotic differentiation; or (3) genes whose levels were significantly changed in late development of mDA neurons (E12-E14) (Fig. 1C) and might be important for late mDA neuron differentiation, including migration and neurite outgrowth.

\section{Laminar structure of the VM}

To better describe the characteristics of the spatial expression pattern of VM-enriched genes, we first defined the morphological organization of the E12 VM using ISH and immunostaining with the aid of established markers for mDA neurons that also showed altered expression in the microarray data. At this point, the VM cells undergo withdrawal from the cell cycle and switch to postmitotic differentiation as described in supplemental Figure S1 E-H (available at www.jneurosci.org as supplemental material). The gene expression pattern demonstrated that the VM in sagittal section may be divided into four layers. Intense signals for both Sox2 and Ki67, which are known to be present in mitotic neuroepithelial cells of the ventricular zone (VZ) (Kele et al., 2006), were detected in the outer stripe of the dorsal layer of the VM (designated layer I) (Fig. $2 A, B$ ). In support of this, doublelabeled staining showed that $\mathrm{Ki}^{+} 7^{+}$cells were devoid of $\beta$ IIItubulin (Fig. $2 B$ ) and TH (data not shown). In contrast, Ngn2 hybridization signals were primarily concentrated in the stripe (layer II) ventral to the VZ (layer I) (Fig. 2C), suggesting that the cell fate of neuroepithelial cells is restricted to $\mathrm{mDA}$ progenitor cells. Moreover, immunoreactivities for Nurr1, a marker for immature mDA neurons, were more intense in the stripe dorsal to the subregion enriched with $\beta$ III-tubulin and TH signals (Fig. $2 D, E)$. The stripe enriched with Nurr ${ }^{+}$cells is designated layer III. Subsequently, cells with prominent $\beta$ III-tubulin and TH signals were considered layer IV (Fig. $2 J$ ).

We found that early B-cell factor (Ebf) protein (revealed by pan-Ebf antibody that recognizes Ebf1-3) was mainly expressed in layers III and IV, with higher level in layer III (Fig. $2 F, G$ ).
However, Ebf1-3 mRNA was mainly expressed in layer III (Fig. $3 K, L$; see Fig. $5 A$ ), in accordance with the findings that Ebf protein is transiently expressed in early postmitotic mDA neurons (Thuret et al., 2004). This suggests that $\mathrm{Ebf}^{+}$cells are mainly immature mDA neurons. These layer III cells showed lower levels of TH, Pitx3, and $\beta$ III-tubulin, although these proteins are dominantly expressed in mature mDA neurons (layer IV) (Fig. $2 \mathrm{~J}, M$ ). Thus, we delineated a four-stage process for mDA neuron development in the VM. These data support the hypothesis that mesencephalic cells undergo continuous differentiation while they migrate ventrally toward the marginal zone. The position of migrating mDA neurons corresponds to the developmental stage of mDA cells that express a specific set of genes. An illustration of this process is shown in Figure $3 R$.

\section{Characterization of the spatial expression pattern of selected VM-enriched genes}

Having defined the "migration-differentiation" model for mDA neuron differentiation in the highly hierarchical structure of the VM, we performed large-scale ISH to validate the spatial expression of 186 genes (162 upregulated and 24 downregulated genes) selected from 1285 differentially expressed genes from the microarray data. The ISH results of 37 representative genes including those described in Figure 1 (bold) are presented in Figures 3 and 5 and supplemental Figure S2 (available at www.jneurosci.org as supplemental material). The VM-enriched genes identified included transcription activators and repressors (e.g., Ebf1-3, Foxp2), cell adhesion molecule close homolog of L1 (Chl1), intracellular signaling molecule Stmn2, transmembrane receptors (e.g., Lrrn3, Dll1), cytoskeleton protein Kelch-like protein 1 (Klhl1), and cyclin-dependent kinase 5 regulatory subunit 1 ( $C d k 5 r 1)$. These have not all been studied in the VM development.

All of these genes were highly expressed in the VM (Fig. 3; see Fig. 5; supplemental Fig. S2, available at www.jneurosci.org as supplemental material), confirming and extending the microarray results. Their hybridization signals were restrictively distributed in the VM with distinct patterns. Fatty acid binding protein 7 (Fabp7) and Tweety homologue 1 (Ttyh1) hybridization signals were detected specifically in the layer I (Fig. $3 A, B$ ). In contrast, $C c n d 2$ and $C d c 2 a$ hybridization signals were highly enriched in layer II, compared with $\mathrm{Ngn} 2$ (Figs. $2 C, 3 F, G$ ). Some gene transcripts, such as $\mathrm{Hmgb2}$, Id3, and Ccnb2, were evenly distributed in both layers I and II (Fig. 3C-E). Moreover, the mRNA expression of $E b f 2, E b f 3, K l h l 1$, and $C d k 5 r 1$ was primarily restricted to layer III cells (Fig. $3 K-N$ ). The best-described mDA neuron marker, Nurr1, was also localized in this subregion (Fig. 3O). Similar to those genes expressed in adjacent layers, expression of Stmn2, Lrrn3, and DII1 was detected in both layers II and III (Fig. $3 H-J$ ), while Chl1 and Foxp2 were expressed in layers III and IV (Fig. $3 P, Q)$. Thus the present study identified a set of VM-enriched genes expressed in distinct pattern, as summarized in Figure $3 R$ (right). These data support the notion that the stepwise DA neuron developmental process is controlled by the combination of a large group of less-restricted genes with a small set of VMenriched genes. Region-specific expression of the VM-enriched genes identified implies their specific roles in distinct stage of VM development.

\section{Determination of the genetic pathways of identified VM-enriched genes}

To further investigate whether the VM restricted genes identified were truly correlated with $\mathrm{mDA}$ neuron development, we used two gene knock-out mouse models: $\mathrm{Ngn}^{-1-}$ and $\mathrm{Lm} \times 1 b^{-1-}$. 


\section{Gene Name}

Description

GenBank ID

Profiles

A. VM early development genes (from E10 to E11)
\begin{tabular}{|l|l|l|l|}
\hline $\begin{array}{l}\text { Esrrg (estrogen-related } \\
\text { receptor } \gamma \text { ) }\end{array}$ & Regulation of transcription & NM_011935 & $\uparrow$ \\
\hline $5730410 E 15 R i k$ & Function unknown & NM_178765 & $\uparrow$ \\
\hline Fgfbp3 & Growth factor binding protein & NM_028263 & $\uparrow$ \\
\hline 2610027 C15Rik & Protein binding unknown function & NM_172145 & $\downarrow$ \\
\hline Bicc1 (bicaudal C homolog 1) & RNA binding protein & NM_031397 & $\downarrow$ \\
\hline $\begin{array}{l}\text { Fabp7 (fatty acid binding } \\
\text { protein 7, brain) }\end{array}$ & Transport & NM_021272 & $\uparrow$ \\
\hline
\end{tabular}
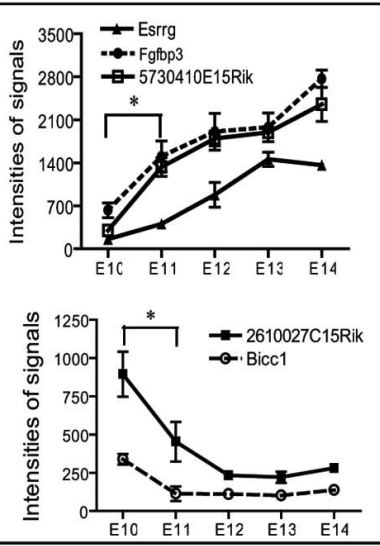

\section{B. VM intermediate development genes (from E11 to E12)}

\begin{tabular}{|l|l|l|l|}
\hline $\begin{array}{l}\text { Sez6I (seizure related 6 } \\
\text { homolog like) }\end{array}$ & Integral to membrane & XM_132255 & $\uparrow$ \\
\hline Nxph1 (neurexophilin 1) & Ligand receptor binding & NM_008715 & $\uparrow$ \\
\hline Ccna2 (cyclin A2) & Cyclin-dependent protein kinase regulator & NM_009828 & $\downarrow$ \\
\hline Bub1 & Protein serine/threonine kinase & NM_009772 & $\downarrow$ \\
\hline Cenpf (centromere protein F) & Regulation of mitotic cell cycle & XM_129658 & $\downarrow$ \\
\hline Ccnd2 (cyclin D2) & Regulation of cell cycle & NM_009829 & $\downarrow$ \\
\hline $\begin{array}{l}\text { Cdc2a (cell division cycle 2 } \\
\text { homolog A) }\end{array}$ & $\begin{array}{l}\text { Regulation of progression through cell } \\
\text { cycle }\end{array}$ & NM_007659 & $\downarrow$ \\
\hline Id3 (inhibitor of DNA binding 3) & Negative regulator of transcription & NM_008321 & $\downarrow$ \\
\hline $\begin{array}{l}\text { Hmgb2 (high mobility group } \\
\text { box 2) }\end{array}$ & DNA replication, regulation of transcription & NM_008321 & $\downarrow$ \\
\hline
\end{tabular}

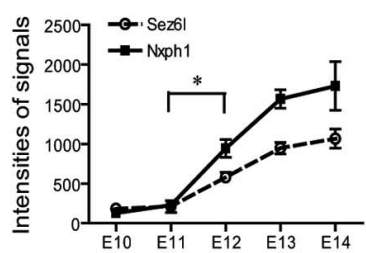

\section{VM late development genes (from E12 to E14)}

\begin{tabular}{|c|c|c|c|c|c|}
\hline B230209C24Rik & Function unknown & NM_177235 & $\uparrow$ & \multirow{4}{*}{ 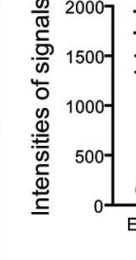 } & \multirow{2}{*}{ 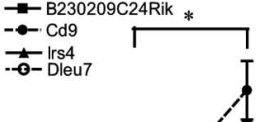 } \\
\hline Cd9 (CD9 antigen) & Cell adhesion & NM_007657 & $\uparrow$ & & \\
\hline Irs4 (insulin receptor substrate 4) & insulin receptor binding protein & NM_010572 & $\uparrow$ & & \\
\hline $\begin{array}{l}\text { Dleu7 (deleted in lymphocytic } \\
\text { leukemia,7) }\end{array}$ & Function unknown & NM_173419 & $\uparrow$ & & $\begin{array}{llll}\text { E10 } & \text { E11 } & \text { E12 } & \text { E13 }\end{array}$ \\
\hline $\begin{array}{l}\text { Cdk5r1 (cyclin-dependent } \\
\text { kinase } 5 \text {, regulatory subunit } 1 \text { ) } \\
\text { (p35) }\end{array}$ & $\begin{array}{l}\text { Cyclin-dependent protein kinase } 5 \\
\text { activator }\end{array}$ & NM_009871 & $\uparrow$ & \multirow{3}{*}{ 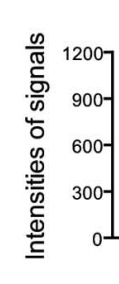 } & 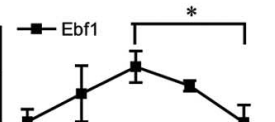 \\
\hline $\begin{array}{l}\text { Chl1 (cell adhesion molecule } \\
\text { with homology to L1CAM) }\end{array}$ & Cell adhesion & NM_007697 & $\uparrow$ & & \\
\hline Ebf1 (early B-cell factor 1) & Transcription factor activator & NM_007897 & $\uparrow 1$ & & $\begin{array}{lllll}\text { E10 } & \text { E11 } & \text { E12 } & \text { E13 } & \text { E14 }\end{array}$ \\
\hline
\end{tabular}

Figure 1. Expression profiles of a subset of VM-enriched genes identified by microarray analysis. A subset of biologically interesting genes that were selected from a larger group of differentially expressed genes is shown. They were classified into three groups $(\boldsymbol{A}-\boldsymbol{C})$, based on expression profiles suggestive of their potential roles during mDA development. The prototypical expression profile of each gene is shown in the right panel. The genes shown in bold are those selected for further analysis. Their graphic gene expression profiles are shown in Figure 3 (except for Ebf1). Arrows indicate upregulated $(\uparrow)$ or downregulated $(\downarrow)$ expression levels of the corresponding genes during mDA neuron development. Values represent means \pm SEM; ${ }^{*} p<0.01$ (ANOVA followed by Newman-Keuls test, $n=3$ ), compared with controls.

Both genes have been reported to be vital for mDA neuron development in different manners (Smidt and Burbach, 2007). Consistent with previous reports (Kele et al., 2006), we found that in addition to abrogated production of $\mathrm{Nurrl}^{+}$and $\mathrm{TH}^{+} \mathrm{mDA}$ neurons in the VM of E12 $N g n 2^{-l-}$ mutants (Fig. $4 A, A^{\prime}$; supplemental Fig. S3a, $a^{\prime}$, available at www.jneurosci.org as supplemental material), large number of cells accumulated in layers I-III rather than migrating to layer IV. This was evidenced by the 

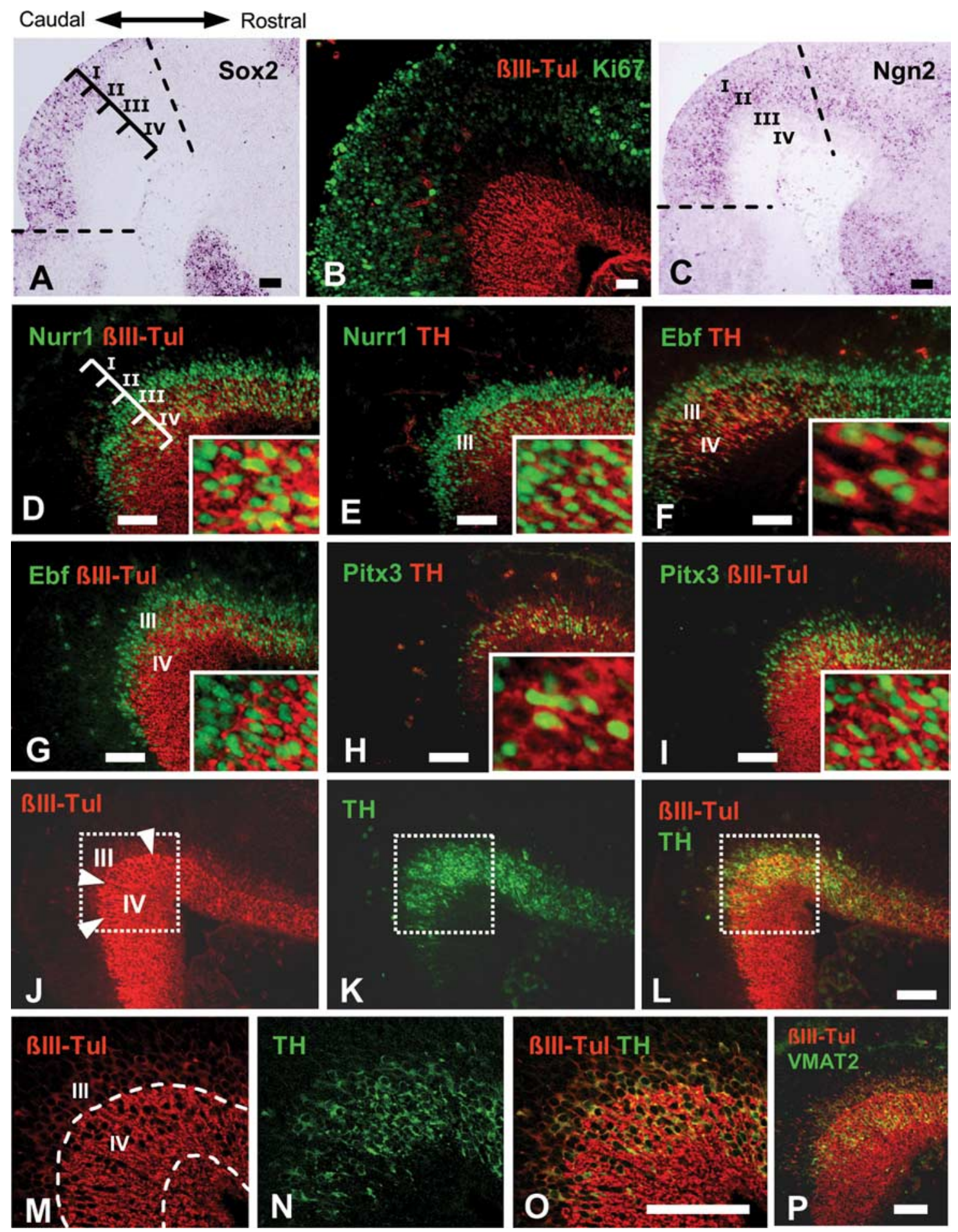

Figure 2. Laminar organization of ventral mesencephalon. In a sagittal section of mouse embryonic brain (E12), the VM may be divided into four layers along the dorsoventral axis based on the restricted expression of various known cell markers. $A, C$, In situ hybridization shows that Sox 2 is expressed primarily in layers I and II, while Ngn2 mRNA is more abundant in layer II than layer I. $\boldsymbol{B}$, Ki67 and $\beta$ III-Tul protein are expressed predominantly in layers I and IV, respectively. D-P, Nurr1 and Ebf are expressed in layer III and Pitx3, TH, and $\beta$ III-Tul are predominantly expressed in layer IV. $\boldsymbol{M}-\boldsymbol{P}$ are enlarged views of the corresponding stippled boxes indicated in $\boldsymbol{J}-\boldsymbol{L}$. Double immunostaining revealed that $\beta \mathrm{III}-\mathrm{Tul}$ is expressed at lower levels in layer III but at higher levels in layer IV. TH and VMAT2 have expression patterns similar to $\beta$ III-Tul. $L$ is a merged photo of $J$ and $\boldsymbol{K} ; \boldsymbol{0}$ is a merged photo of $\boldsymbol{M}$ and $\boldsymbol{N}$. Arrows in $\boldsymbol{J}$ indicate layers III/IV and layer IV/mesencephalic marginal strip boundaries. Scale bars, $100 \mu \mathrm{m}$.

fact that the density of $L m \times 1 a^{+}$or $L m \times 1 b^{+}$cells, indicating a laminar structure in the VM, was increased remarkably in layers I-III, in particular layer III, with an absence in layer IV compared with wild-type mice (Fig. $4 B-C^{\prime}$; supplemental Fig. S3c, $c^{\prime}$, available at www.jneurosci.org as supplemental material). These data are in accordance with a previous report (Andersson et al., 2006) and support the hypothesis that Ngn2 controls the differentiation and migration of mDA neurons, indicating that $\mathrm{Ngn}^{-/-} \mathrm{mu}-$ tants are thus very useful for determining potential genetic associations between $\mathrm{Ngn} 2$ and selected genes. 


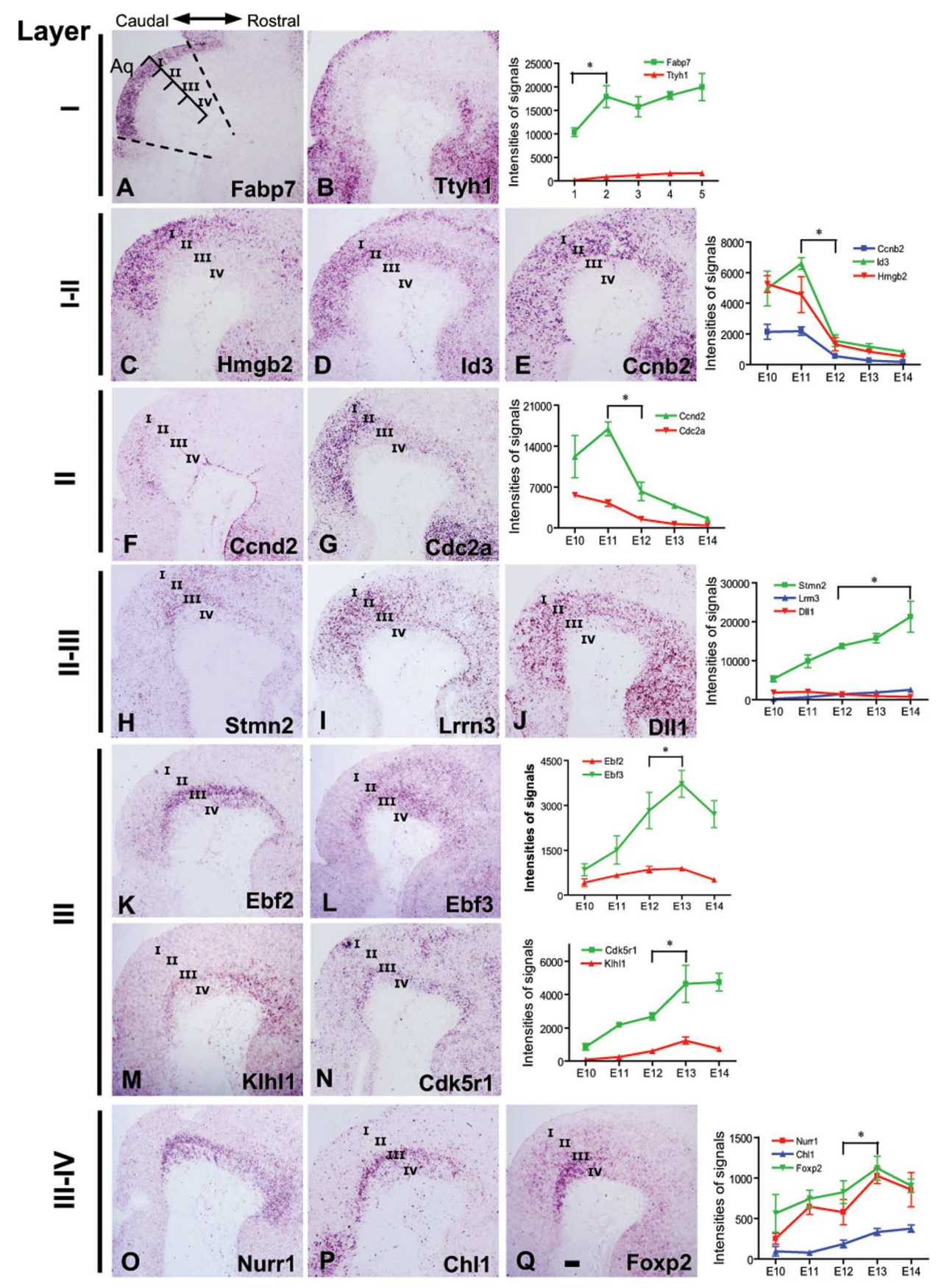

$\mathbf{R}$

Layer / Stage

I Neuroepithelial
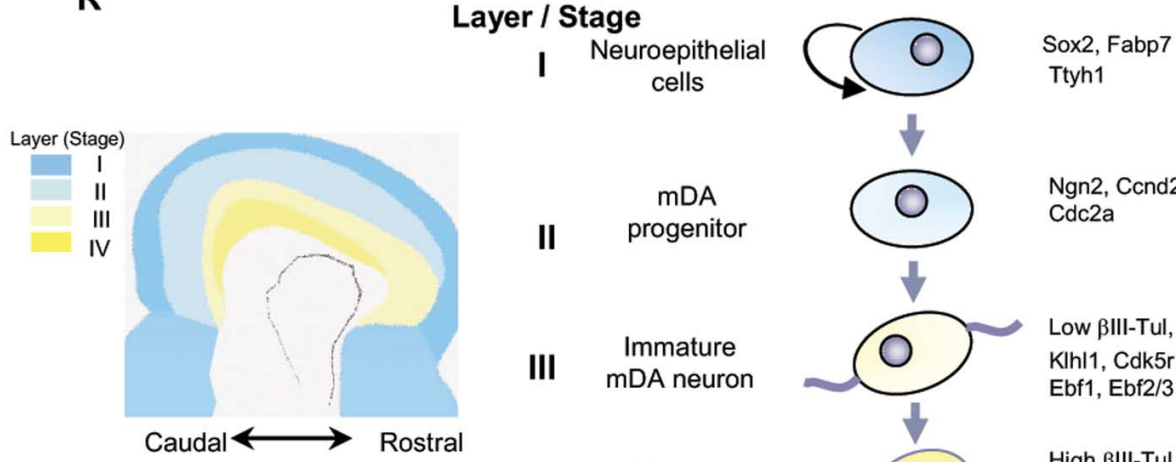

cells

Sox2, Fabp7

Ttyh1

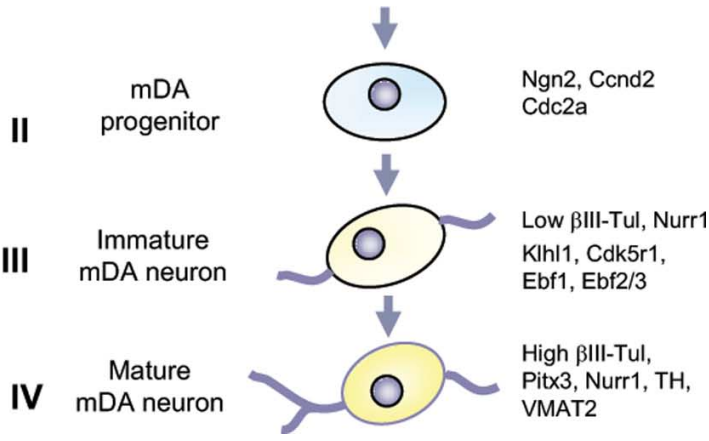


Using Ngn2 ${ }^{-/-}$mutant mice, we examined the expression of several transcription activators and repressors (Ebf, Foxp2, Pbx3, Npas3, Fabp7, Hmgb2, Id3, and Klf7) that we identified as VMenriched genes in the present study. We found that Ebf immunosignals were absent in $\mathrm{Ngn} 2^{-1-}$ mutants at E12 (Fig. $4 D, D^{\prime}$ ). ISH analysis showed that Ebf1 mRNA was also undetectable in these mutants (data not shown). Conversely, expression of Ebf protein (Fig. $4 K, K^{\prime}$ ) and Ebf1-3 mRNA (supplemental Fig. S3B, available at www.jneurosci.org as supplemental material) remained unchanged in $L m \times 1 b^{-1-}$ mutants. Additionally, Foxp2, $\mathrm{Pbx3}$, and Npas3 expression was absent in the $\mathrm{VM}$ of $\mathrm{Ngn} 2^{-1-}$ mutants (Fig. $4 E-G^{\prime}$ ). In contrast, expression of Fabp7, Hmgb2 (supplemental Fig. S3A, available at www.jneurosci.org as supplemental material), $I d 3$, and $K l f 7$ (data not shown) in the VM was not markedly altered in the absence of Ngn2 activity. Moreover, $L m \times 1 b$ depletion did not significantly affect expression of any of these transcription activators and repressors in E12 VM (Fig. 4, right; supplemental Fig. S3B, available at www.jneurosci.org as supplemental material), despite the fact that aromatic L-amino acid decarboxylase (AADC) (Fig. 4I, I') and Pitx3 (supplemental Fig. S3A, available at www.jneurosci.org as supplemental material) expression was diminished. Together, these results suggest that Ebf, Foxp2, $P b \times 3$, and Npas 3 are likely to be involved in mDA neuron development under the control of $\mathrm{Ngn} 2$. A sketch of genetic pathways is shown in Figure 40.

Together, these data demonstrated that a set of VM-enriched genes with specific spatial and temporal expression characteristics was identified. It is likely that these genes play important roles in the respective phases of the differentiation and migration of mDA neurons.

\section{Ebf1 is expressed primarily in immature mDA neurons}

To begin to understand the roles of identified VM-enriched genes, we selected $E b f 1$ for further study, because Ebf1 was highly expressed in the VM with a peak level at E12 (Fig. 1), when mDA neural progenitors withdraw from mitosis and start to differentiate/migrate to establish nigrostriatal projections. These results suggest that Ebf1 may have an important and novel function in these processes. To test this hypothesis, we characterized more precisely the expression pattern of Ebf1 in the VM. ISH analysis showed that Ebf1 mRNA was predominantly expressed in layer III of E12 VM, with much low level in layer IV (Fig. $5 A-D)$ ). In contrast, $T H$ and vesicle monoamine transporter 2 (VMAT2) were expressed in both layers III and IV with higher levels in layer IV (Fig. 5 B,C). Combined Ebfl ISH with TH immunostaining revealed that some $E b f 1^{+}$neurons in layer III expressed TH at

$\leftarrow$

Figure 3. Regional specific expression of genes identified from the microarray analysis of the VM (A-Q). In situ hybridization in sagittal sections of the mouse embryonic brain (E12) shows restricted expression of 17 genes selected in the distinct layers of VM. Dashed lines indicate the position of the VM. Scales indicate the four-layer laminar structure in the VM. $\boldsymbol{A}-\boldsymbol{E}$, Ttyh1 and Fabp7 are expressed in layer I, while $H m g b 2$, Id3, and C(nb2 are predominantly expressed in layers I and II. F, G, Ccnd2 and Cdc2a show restricted expression in layer II. $\boldsymbol{H}$-J, Stmn2, Lrrn3, and DII1 are expressed in layer II/III cells. $\boldsymbol{K}-\boldsymbol{N}, E b f 2, E b f 3, K / h / 1$, and $C d k 5 r 1$ are specifically expressed in layer III. $\mathbf{0}-\mathbf{Q}$, Nurr1, Chl1, and Foxp2 mRNA are detected in layers III and IV. The prototypical expression profile of each gene is shown in the right panel. The data represent means \pm SEM of three independent experiments. Asterisks indicate statistical significance between two adjacent time points $(p<0.05)$. Scale bar, $100 \mu \mathrm{m}$. $\boldsymbol{R}$, Schematic representation of a potential model for the generation of $\mathrm{mDA}$ neurons from neuroepithelial cells. The model shows a four-layer laminar organization in the VM (left), which corresponds to a four-stage $\mathrm{mDA}$ neuron development (right). Some genes that are expressed by developing mDA cells, identified by microarray analysis, are indicated at right.
E13 (Fig. 5D,E). A few $\mathrm{Ngn}^{+}{ }^{+}$cells also coexpressed Ebf (data not shown). These results indicate that Ebfl is highly expressed in immature mDA neurons, compatible with the four-layer laminar model shown in Figure 3R. At E14 and E15, Ebf immunosignals were colocalized with $\mathrm{TH}$ in the large number of $\mathrm{mDA}$ neurons in the SN primordium (Fig. $5 F, G, I$ ). Indeed, Ebf1 hybridization signals were also detected in the SN primordium at E15 (Fig. $5 \mathrm{H}$ ) but not at postnatal day 0 (P0) (data not shown). Thus, the period of transient Ebf1 expression in embryonic mDA neurons coincided with the early period of SN formation. Thus, Ebf1 is not required for the control of cell proliferation and $\mathrm{mDA}$ neuron generation in the VM.

\section{Impaired formation of the substantia nigra pars compacta in Ebf $1^{-1-}$ mutants}

Surprisingly, we found that $E b f 1^{-1-}$ mice exhibited severe impairment in the formation of the substantia nigra pars compacta (SNc) during development. At E15, the number of $\mathrm{TH}^{+}$cells migrating diagonally upward was remarkably reduced in the Ebf1 ${ }^{-1-}$ mutants. The deficiency was persistent even at later stages $\mathrm{E} 16$ and $\mathrm{P} 0$ (Fig. $6 A-H$ ). In contrast, the SNc primordium appeared normal at E13 and E14 in Ebf1 ${ }^{-1-}$ mutants (data not shown). At P0, the number of $\mathrm{TH}^{+}$cells in the $\mathrm{SNc}$ was only $54 \%$ of the control (mutants: $499.3 \pm 11.4$ vs control: $919.7 \pm 40.1$, $n=3$ for each, $p<0.05$ ). Remarkably, only $13 \%$ of $\mathrm{TH}^{+}$cells migrated to the lateral part of the SN (SNL) compared with the control (mutants: $11.5 \pm 3.1$ vs control: $121.0 \pm 8.9, n=3$ for each, $p<0.05$ ) (Fig. $6 I-L$ ), although the size and shape of the P0 $\mathrm{Ebfl}^{-1-}$ mutant brain were not markedly altered compared with wild-type mice, as illustrated in Figure 7, $A$ and $B$. The disorganization in the $\mathrm{SN}$ remained in the adult; the distribution of $\mathrm{mDA}$ neurons was severely perturbed with a complete absence of $\mathrm{TH}^{+}$ neurons in the SNL and a scrambled structure in the substantia nigra pars reticulata $(\mathrm{SNr})$ (Fig. $6 \mathrm{M}-\mathrm{P})$. Interestingly, the deficiency was observed in both the rostral and intermediate parts of the SNc (Fig. 6), from which the DA neurons send their projections to the striatum constituting the mesostriatal pathway. In contrast, $\mathrm{TH}$ expression in the caudal part of the SNc was not markedly altered in either embryos or neonates (data not shown).

To determine whether Ebf1 might act as a postmitotic selector of SN neuronal identity and therefore SN neurons might change their identity and acquire that of VTA neurons in the absence of $E b f 1$, we analyzed the expression of markers preferentially expressed in the SN or VTA of young DA neurons, such as Girk2 (for SN) and calbindin (for VTA), to assess whether there is a change in the proportion of DA neurons immunoreactive for these markers between mutant and control mice. The number of calbindin ${ }^{+}$neurons in the VTA was not significantly changed in Ebf1-null mice at P0 (control: $1026 \pm 30.56$ cells vs Ebf1 $^{-1-}$ mutants $1050 \pm 53.69$ cells, 4 wild-type and 4 mutants, $p>0.05$ ) (Fig. 7C,D). Moreover, those DA neurons that failed to migrate to the SNc were Girk2 positive (data not shown). These data suggest that $E b f 1$ is specifically required for the development of nigral but not VTA DA neurons.

To determine whether the disorganized SN in Ebf1 $1^{-1-} \mathrm{mu}-$ tants is a secondary effect of a distorted brain following Ebf1 depletion, we compared the VM architecture of Ebf1 ${ }^{-1-}$ mice with that of wild-type littermates. The entire VM laminar structure labeled with $\mathrm{Lm} x \mathrm{Ia}$ antibody in E12 $\mathrm{Ebf1}{ }^{-/-}$mice was normal (Fig. 8 A). Similarly, at P0, the general structural arrangement of nuclei in the midbrain, revealed by Nissl staining, was not markedly altered between mutants and controls (data not shown). Oculomotor nuclei that are located laterally to the SN, 


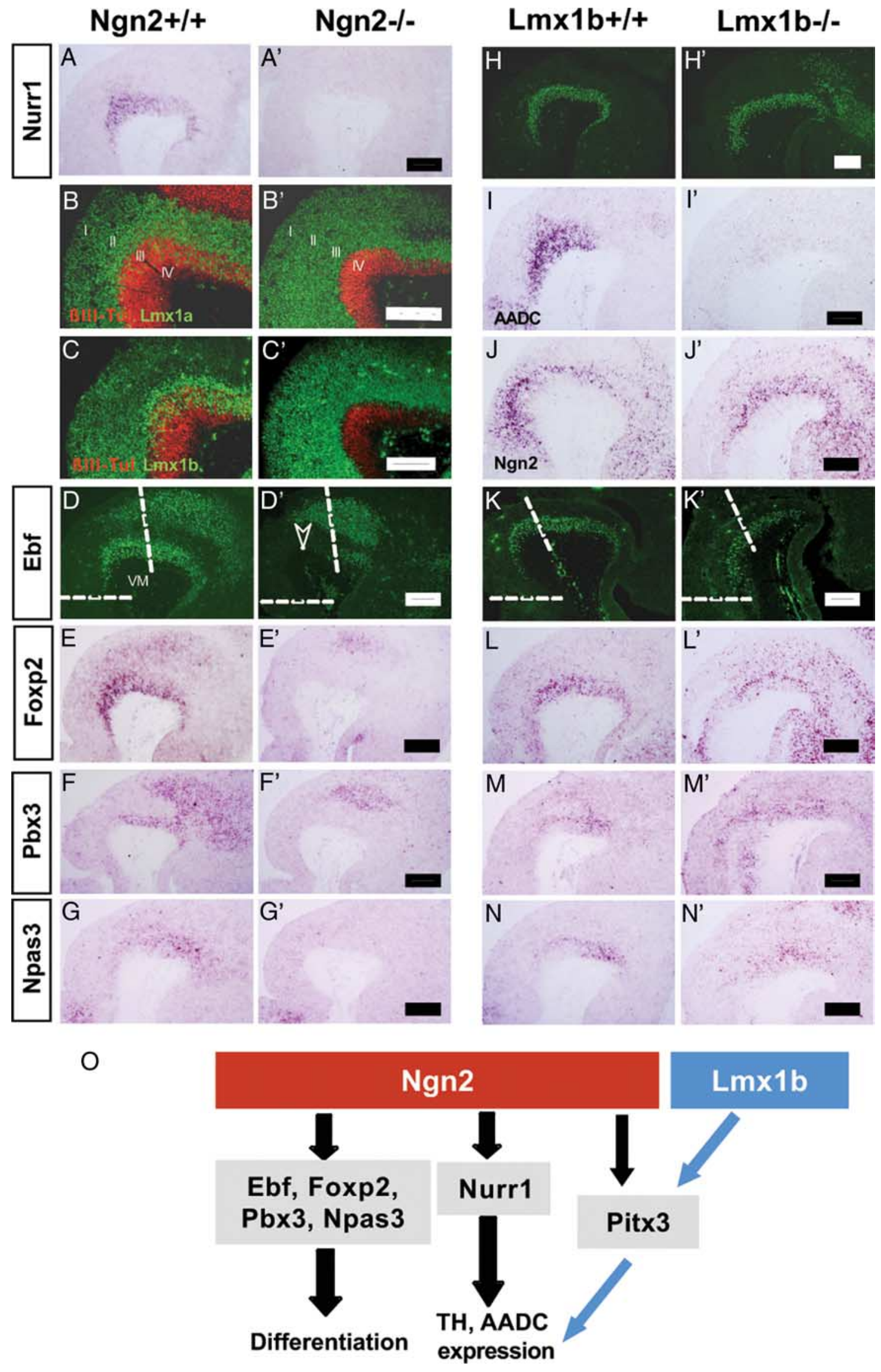


representing a non-DA brain structure adjacent to the SN, were largely identical between mutant and wild-type mice, as revealed by Isl1 immunostaining in $\mathrm{P} 0$ Ebf1 ${ }^{-1-}$ mutants (data not shown). Together, these data suggest that Ebf1 depletion in embryos and neonates does not lead to substantial disorganization of neuronal lamination in the VM or the neighboring brain structure.

\section{Lack of mDA neurons in the $\mathrm{SNc}$ of} $E b f 1^{-/-}$mutants results from impaired diagonal upward migration from the VTA We then asked whether reduction of $\mathrm{mDA}$ neurons in the Ebf1 ${ }^{-1-}$ mutants was due to loss of $\mathrm{TH}$ expression or apoptosis of mDA neurons in the SNc. Pitx3 immunostaining, which specifically labels $\mathrm{mDA}$ neurons (Nunes et al., 2003), was used to visualize these cells. The advantage of this staining is that it allows visualization of highly compact $\mathrm{mDA}$ neurons individually, thus facilitating their quantification. The number of Pit $3^{+}$cells was reduced to $53.7 \%$ in the $\mathrm{SNc}$ of $E b f 1^{-1-}$ mutants compared with controls at P0 (mutants, $618.24 \pm 64.2$ vs control: $1151.0 \pm 55.1$, $n=4$ for each, $p<0.05)$. In contrast, the number of Pitx $3^{+}$cells in the VTA was slightly increased (mutant: $4052 \pm 279.0$ vs control: $3656.75 \pm 296.0, n=4)$, although there was no change in the total number of Pitx $3^{+}$cells in the VM (including the SN, VTA, and those along the midline) (mutant: $4669.0 \pm 274.5$ vs control: $4745.0 \pm 409.4, n=4)$. The terminal deoxynucleotidyl transferase-mediated dUTP-biotin nick-end labeling (TUNEL) staining showed no alteration in apoptotic cells in either $E b f 1^{-1-}$ mutants or wildtype embryos at E14, E15, E16, and P0 (data not shown). These data suggest that in Ebf1 ${ }^{-/-}$mutants, the lack of mDA neurons that are destined for the $\mathrm{SNc}$ does not result from a deficiency in their neurogenesis or survival.
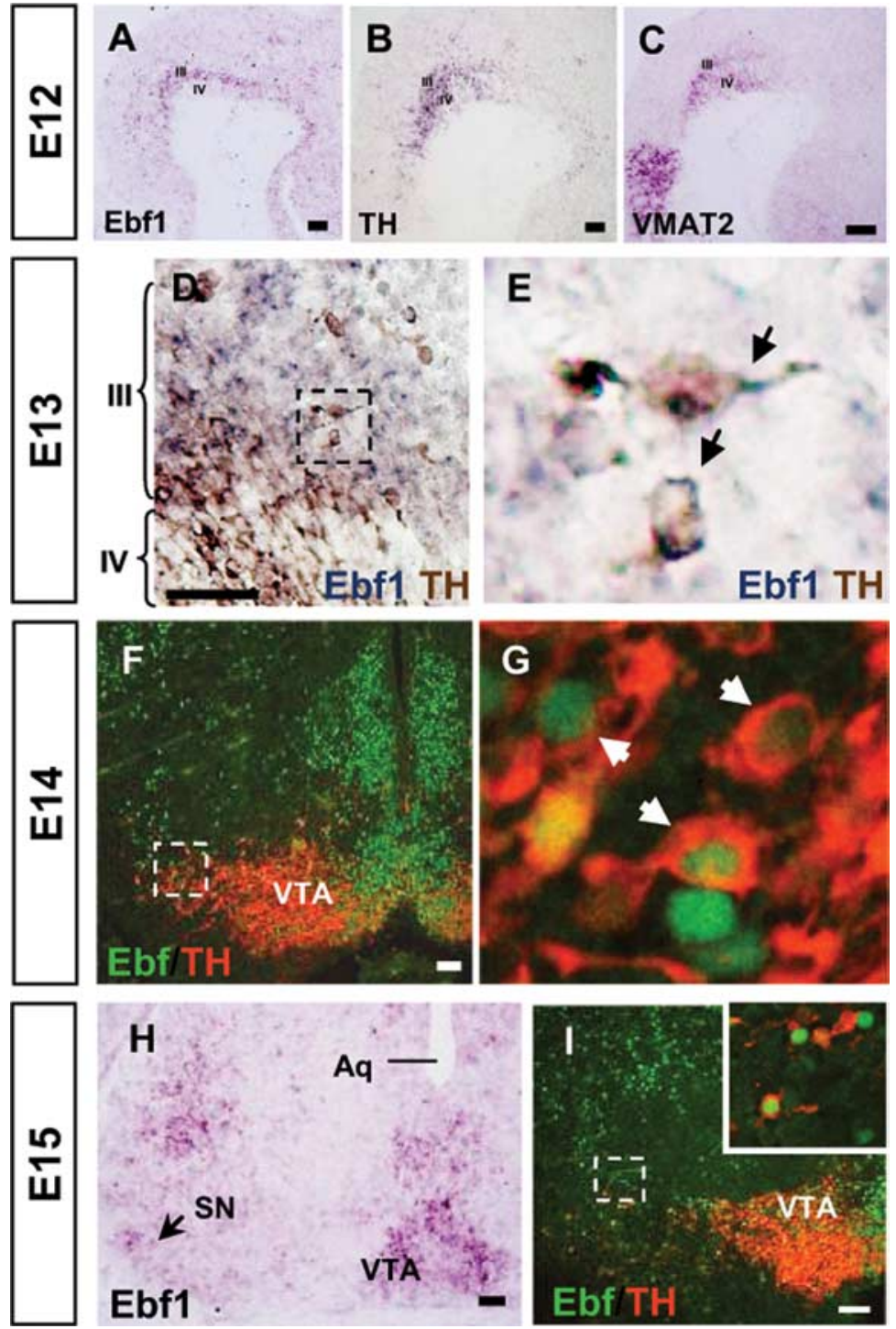

Figure 5. Expression of $E b f 1$ in the VM. $\boldsymbol{A}-\boldsymbol{E}$, In situ hybridization reveals that Ebf1 is expressed specifically in layer III $(\boldsymbol{A})$, whereas TH and VMAT2 mRNA are predominantly expressed in the layer IV of E12 VM in a parasagittal section $(\boldsymbol{B}, \boldsymbol{C}) \boldsymbol{D}, \boldsymbol{E}$, Prominent Ebf1 hybridization signals (purple) are detected primarily in layer III at E13. Combined in situ hybridization and immunostaining show that Ebf1 hybridization signals (purple) are colocalized with TH immunoreactivity (brown) in some of layer III cells. III, Layer III; IV, upper part of layer IV. E is a magnified image of the stippled box shown in D. Arrows indicate double-labeled $\mathrm{mDA}$ neurons in layer III. $\boldsymbol{F}, \boldsymbol{G}$, Double-labeled immunofluorescence photomicrographs showing coexpression of Ebf and TH (indicated with arrows) in E14 embryos in a coronal section. $\boldsymbol{H}, \boldsymbol{I}$, In a coronal section of an E15 mouse embryo, Ebf1 hybridization signals are detected in both the SN and VTA $(\boldsymbol{H})$. Double-labeled immunofluorescence shows that at E15 when the SN is being formed, a few $\mathrm{TH}^{+}$neurons migrating toward the SN express Ebf protein (I). Aq, Aqueduct. Scale bars, $100 \mu \mathrm{m}$.
Figure 4. VM-enriched genes Ebf, Foxp2, Pbx3, and Npas3 are regulated by Ngn2. Expression of these genes in the VM was determined in E12 Ngn2 and Lmx1b null mutant mice. Established mDA neuron markers Nurr1, $L m \times 1 a, \operatorname{Lm} \times 1 b$, and $A A D C$ served as positive controls. $\boldsymbol{A}, \boldsymbol{A}^{\prime}$, Deficiency of Ngn2 results in depletion of Nurr1. $\boldsymbol{B}-\boldsymbol{C}^{\prime}$, Double immunofluorescence shows that $L m \times 1 a^{+}$or $L m \times 1 b^{+}$cells accumulate in layers I-III, suggesting that their ventral migration is arrested in the absence of $\mathrm{Ngn} 2 . \boldsymbol{D}-\boldsymbol{G}^{\prime}$, Immunostaining $\left(\boldsymbol{D}, \boldsymbol{D}^{\prime}\right)$ or in situ hybridization $\left(\boldsymbol{E}-\boldsymbol{G}^{\prime}\right)$ shows that Ebf, Foxp2, Pbx3, and Npas3 are not expressed in the absence of $\mathrm{Ngn} 2$, whereas their expression is normal in $L m \times 1 b^{-1-}$ mutants $\left(\boldsymbol{J}-\boldsymbol{K}^{\prime}\right) . \boldsymbol{H}-\boldsymbol{N}^{\prime}, \mathbf{L m} \times 1 b$ depletion does not alter Nurr1, Ngn2, Ebf, Foxp2, and Npas3 expression, whereas AADC expression disappears. Note that in $L m \times 1 b^{-/-}$null mutants, the mesodiencephalic structure is shrunken and severely distorted (right). The sagittal views of the brain sections are not always well matched between mutants and controls. Scale bars, $200 \mu \mathrm{m}$. Data represent at least three independent experiments. Dotted lines indicate diencephalon/mesencephalon and mesencephalon/metencephalon boundaries. $\mathbf{O}$, Downstream molecules that are regulated by $N g n 2$ and $L m \times 1 b$ are shown.
Interestingly, the effect of Ebf1 depletion on mDA neuron development appeared to be specific to nigral DA neurons. Only the projection terminals of $\mathrm{mDA}$ neurons in the striatum were dramatically reduced in Ebf1 ${ }^{-1-}$ mutants, whereas those in the accumbens nucleus were not altered (Fig. 7E,H). This is consistent with the observation that the number of Pitx $3^{+}$neurons in homozygous mutant mice was modified in the $\mathrm{SNc}$ but not the VTA.

\section{Both radial and tangential fibers are unaffected by the absence of $E b f 1$}

To gain insight into the defective migration of mDA neurons in Ebf1-deficient mice, we examined the structure of both radial and tangential fibers in the VM. It was observed that Nestin ${ }^{+}$radial glial fibers extended from the VZ to the pial surface of the VM at 


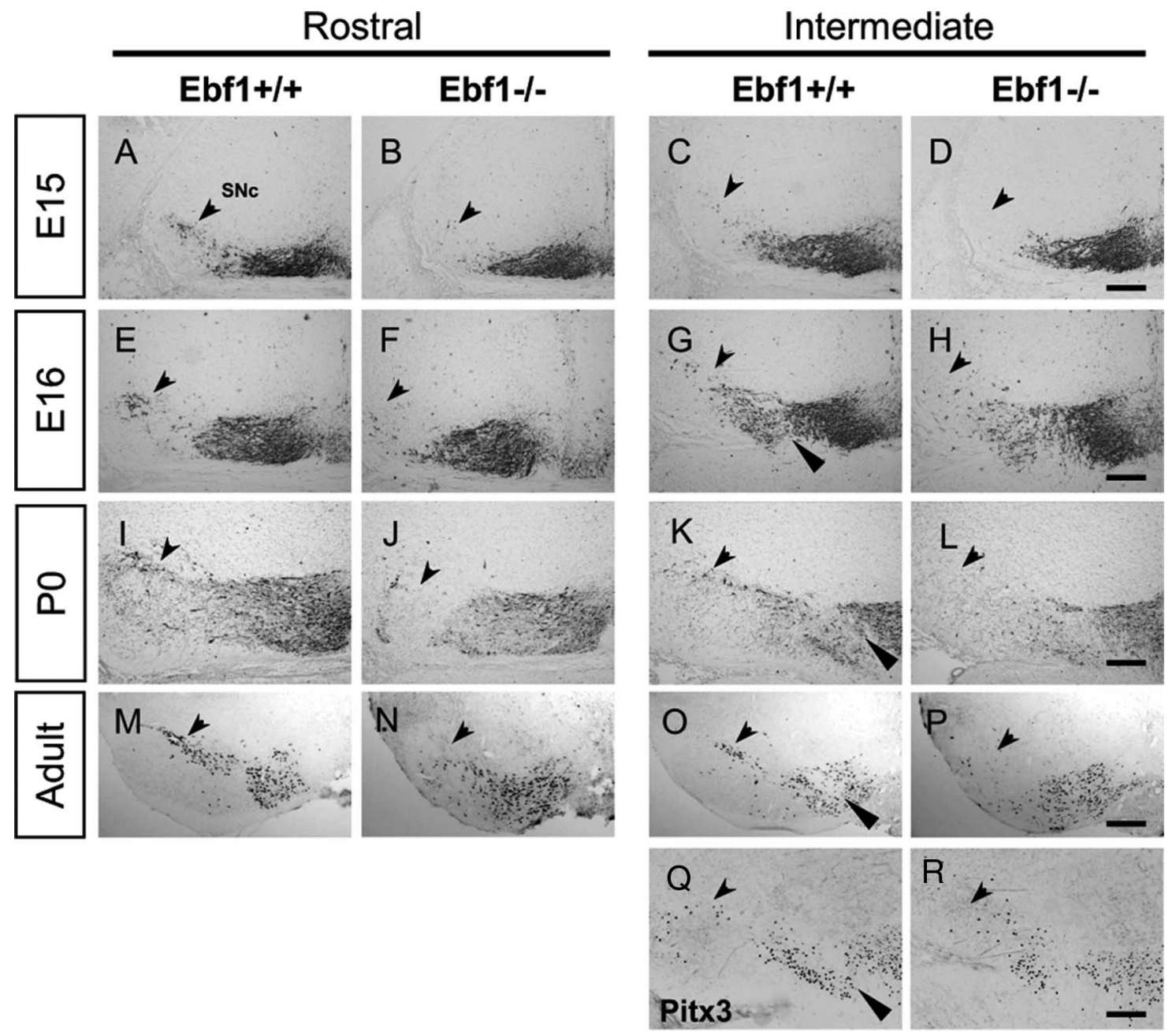

Figure 6. Deficiency in the formation of the SNc in Ebf1 ${ }^{-1-}$ mutants. The distribution patterns of mesencephalic DA neurons between Ebf1 ${ }^{-1-}$ mutants and wild-type mice are compared at various stages during development. Immunohistochemistry shows that TH expression pattern is altered in the SNc of embryonic and postnatal Ebf1 ${ }^{-1-}$ brain $(\boldsymbol{A}-\boldsymbol{L})$. In situ hybridization analysis shows that Ebf1 depletion results in a dramatic loss of $\mathrm{TH}^{+}$neurons in the SNc of adult Ebf1 ${ }^{-1-}$ mice compared with wild-type mice $(M-P)$. Arrows indicate the location of the SN. Arrowheads indicate the medial terminal nucleus of the accessory optic tract (MTN), which separates the SN and VTA. $\boldsymbol{Q}$ and $\boldsymbol{R}$ show the distribution pattern of Pitx $3^{+}$cells in the Ebf1 ${ }^{-/-}$mutants and wild-type mice at P0. Scale bars, $200 \mu \mathrm{m}$.

E12 (Shults et al., 1990) and were arrayed in a similar pattern between $\mathrm{Ebf1}^{-1-}$ mutants and wild-type embryos (Fig. 8A). Moreover, the tangential fibers revealed by $\mathrm{L} 1$ antibody (Ohyama et al., 1998) extended normally in the medial part of the VM through the middle toward the lateral part in $E b f 1^{-1-}$ mutants as those in wild-type mice. In the middle and lateral parts of the VM, L1-bearing fibers appeared as short and clustered structures, and the migrating $\mathrm{TH}^{+}$neurons were alongside these fibers (Fig. $8 \mathrm{~B}$ ).

\section{Discussion}

In the present study, we describe a molecular characterization of the VM between E10 and E14, a discrete yet critical window for mDA neuron generation, differentiation, migration, and circuitry formation. We identified a set of VM-enriched genes that were expressed in distinct subpopulations of VM cells. Their elaborate spatial expression patterns strongly suggest that $\mathrm{mDA}$ neuron development is a highly complex but coordinated process. This highlights the importance of correct mDA neuron development, as demonstrated by a deficiency of the layer IIIspecific gene Ebf1 resulting in impaired formation of the SNc.

\section{Systematic analysis of VM development using} microarray analysis

Neural development is a fast process with rapid dynamic changes in gene expression levels. Previous studies on neural development using high-throughput microarrays have often been performed at multiple time points with relatively long intervals. We hypothesized that frequent sampling is a key for successful molecular dissection of VM development. Thus one of the most remarkable features of the present study is the genome-wide DNA microarray analysis of the VM dissected on daily basis from E10 to E14. Indeed, by adopting this strategy, we were able to establish a more subtle map of gene expression, potential regulation, and molecular interactions during mDA neuron development. For example, we identified reelin-based signaling pathway governing neural migration in the VM (data not shown). Moreover, the direct and precise microdissection of mouse VM performed in the present study provides sufficient tissue for microarray analysis without RNA amplification. Thus subsequent gene profiling accurately reflects in vivo gene expression. This strategy therefore significantly enhances the probability of iden- 
Ebf1+/+

A
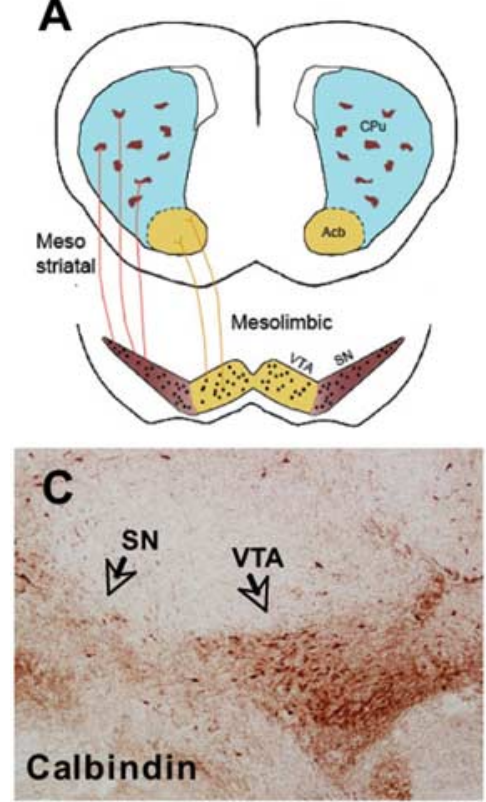

E
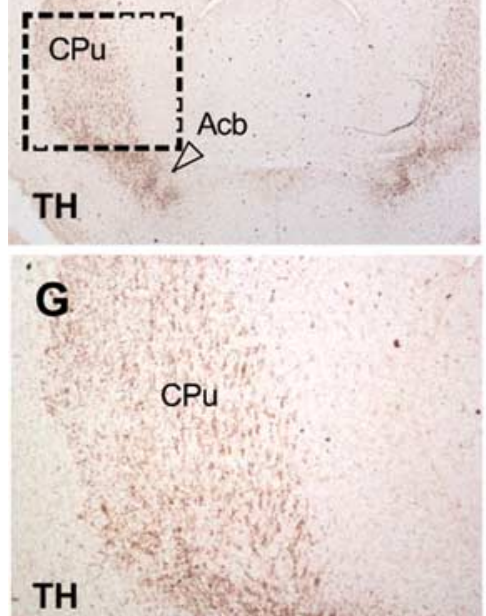

Ebf1-/-
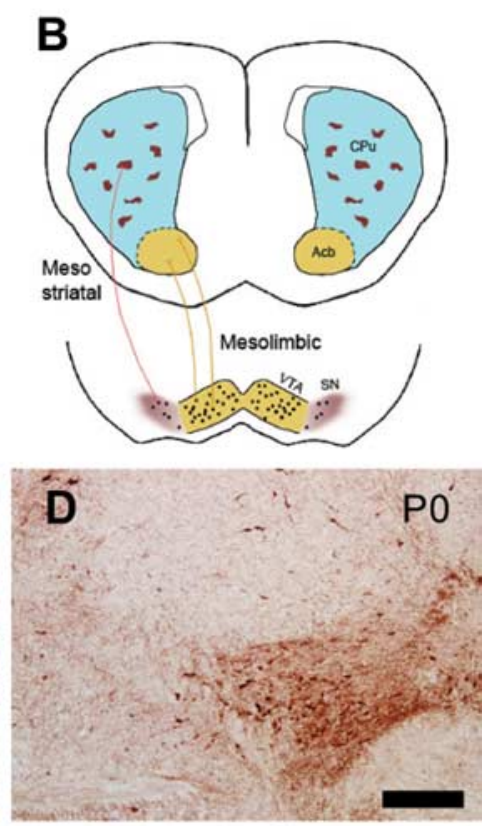

$\mathbf{F}$

E16

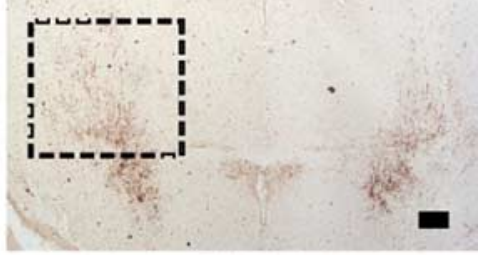

H

E16

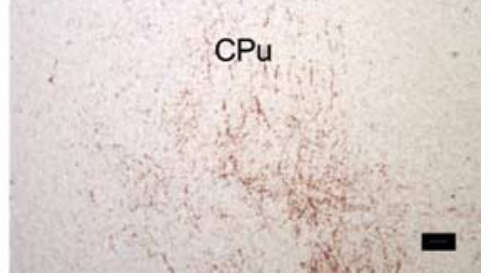

Figure 7. $A, B$, Schematic representations of coronal views of the basal forebrain in wild-type and $E b f 1^{-1-}$ mutants, respectively, showing the location of DA neurons in the SNc (black dots in brown colored areas) and their axonal projections (mesostriatal pathway in red) toward the striatal patches (dark brown) in the caudate-putamen nucleus ( $\mathrm{CPu}$, blue). The DA neurons in the VTA send their projections to the nucleus accumbens (Acb), constituting the mesolimbic pathway (yellow). $A$ and $B$ were adopted with permission from Yue et al. (1999) with modifications. C, D, Calbindin immunostaining shows no marked changes in calbindin ${ }^{+}$ cells in the VTA. Mispositioned mDA neurons express Girk2 at P30 in the absence of Ebf1.E, $\boldsymbol{F}$, TH immunostaining reveals that the density of $\mathrm{TH}^{+}$terminals in the $\mathrm{CPu}$, but not in the Acb, was markedly reduced in Ebf1 ${ }^{-1-}$ mutants at $\mathrm{E} 16$ compared with wild-type mice. $\boldsymbol{G}$ and $\boldsymbol{H}$ are magnified fields indicated by stippled boxes in $\boldsymbol{E}$ and $\boldsymbol{F}$. Scale bars, $200 \mu \mathrm{m}$.

tifying genes that truly play a role in regulation of $\mathrm{mDA}$ neuron development.

\section{Laminar organization of the VM: functional implications}

One of remarkable features of the developing VM presented here is that $\mathrm{mDA}$ development is concomitant with cell migration. Their differentiation is closely associated with their physical location in the subregions of the VM. Cells differentiate to a specific stage only when they migrate to the corresponding subregion.
Thus developing mDA cells exhibit clear morphological laminar features-a fourlayered structure, supporting the hypothesis that the generation of mDA neurons follows a stage-by-stage process governed by a genetic program (Ang, 2006). Importantly, we found that cells in each layer express a subset of specific genes (Figs. 1, 3), which are apparently associated with distinct aspects of their development, such as differentiation, migration, and axonal pathfinding. For example, disruption of the layer II-specific gene $N g n 2$ results in arrest of cell migration accompanied by a failure of cell differentiation (loss of $\mathrm{TH}^{+}$ neurons). Ebf1 depletion leads to retardation of mDA neurons in the marginal zone. These findings highlight the significance of the laminar structure of the VM, which shows unique morphological and, more importantly, functional features. Interestingly, this model for mDA development is distinct from neural development in some other brain regions, such as the cerebrocortex, which is a highly organized, six-layered structure. Unlike developing mDA cells, the layering of cortical neurons is established in an inside-first, outsidelast manner (Kandel et al., 2000). Pyramidal cells in each layer of the cortex are mature neurons expressing specialized properties, whereas in the VM only layer IV cells are mature DA neurons (Molyneaux et al., 2007). Thus the genetic program controlling $\mathrm{mDA}$ development is distinct from that in other brain regions.

\section{Identification of VM-enriched genes}

Our data revealed that genes in three categories were identified during $\mathrm{mDA}$ neuron development: (1) genes that regulate general events during brain development, including cell fate determination and differentiation (e.g., Fabp7); (2) genes that are restricted to the VM and may be involved in multiple aspects of mDA neuron differentiation, survival, and migration (e.g., Ngn2, Ccnd2); and (3) genes that were highly restricted to a single layer of the developing VM and played a crucial role in a specific aspect of mDA neuron development (e.g., Ebf1). Many of these genes have not been previously studied in MDA neuron development and thus are novel components important for this process.

We found some genes that showed a gradient expression pattern across the different layers. For example, Hmgb2, Id3, and $C \mathrm{cnb2}$ are localized in layers I and II, whereas expression of Stmn2, Lrrn3, and Dll1 is found in layers II and III, suggesting that they are expressed by different subtypes of developing mDA cells. These results indicate that different subtypes of developing mDA cells use similar genetic machinery to drive their growth and differentiation. These genes likely work together in a variety of 
A
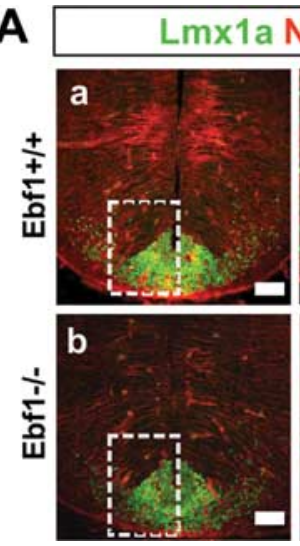

B
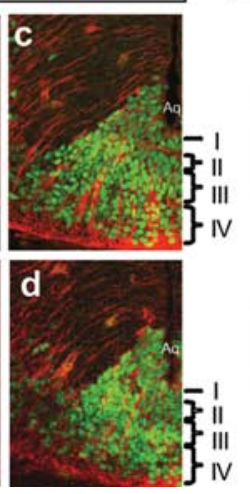

\section{Medial VM}
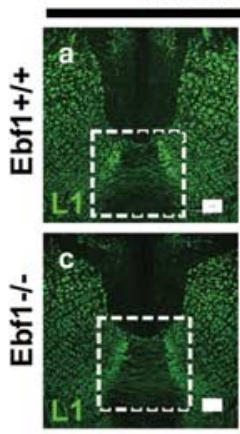
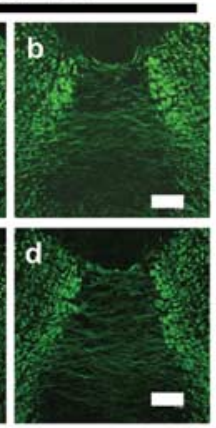

Lateral VM

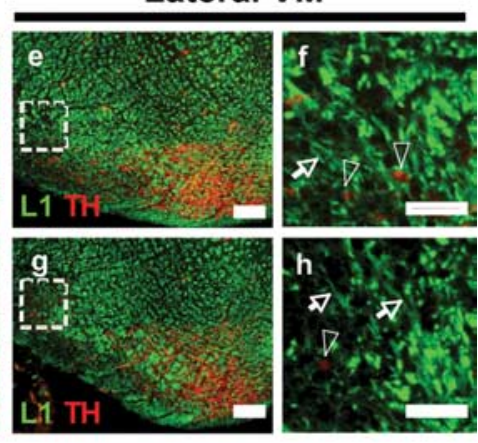

Figure 8. Both radial glial fibers and tangential fibers are well conserved in the absence of Ebf1.A, Photomicrographs showing that the radial glial fibers stained with nestin antibody and laminar organization revealed by $L m \times 1 a$ immunofluorescence are well formed in E12 Ebf1 ${ }^{-1-}$ mutants $(n=2)$. c and $\boldsymbol{d}$ are magnified images of the stippled boxes in $\boldsymbol{a}$ and $\boldsymbol{b}$. I-IV indicate layers I-IV. $\boldsymbol{B}$, The distribution profile of tangential fibers stained with L1 antibody in the medial $(\boldsymbol{a}-\boldsymbol{d})$ and lateral $(\boldsymbol{e}-\boldsymbol{h})$ parts of the VM of E15 Ebf1 ${ }^{-/-}$mutant embryos is very similar to that in wild-type mice $(n=3) . \boldsymbol{b}, \boldsymbol{d}, \boldsymbol{f}$, and $\boldsymbol{h}$ are magnified views of the stippled boxes indicated in $\boldsymbol{a}, \boldsymbol{c}, \boldsymbol{e}$, and $\boldsymbol{g}$, respectively. Arrows indicate tangential fibers in green. Arrowheads indicate TH ${ }^{+}$neurons in red. Scale bars, $150 \mu \mathrm{m}$.

combinations to provide a basic genetic platform upon which different aspects of VM development are tightly controlled.

\section{A biological function for layer III-specific gene $\boldsymbol{E} \boldsymbol{b} \boldsymbol{f} \mathbf{1}$}

Having characterized the spatial and temporal expression pattern of the layer III-specific genes, we found remarkable defects in $E b f 1^{-1-}$ mutant mice in the positioning of nigral DA neurons and their axonal projections to the striatum. mDA neurons in $E b f 1^{-1-}$ mice failed to migrate to the $\mathrm{SNc}$, from which they send their projections to the striatum. Ebf1 belongs to Ebf/Olf1 gene family, which was first identified as a family of transcription factors implicated in mouse B-lymphocyte differentiation. Ebf1 has been implicated in the control of neuronal differentiation, migration, and axonal outgrowth in non-DA systems (Garel et al., 2000; Garcia-Dominguez et al., 2003; Wang et al., 2004). In basal forebrain, $E b f 1$ is specifically involved in striatal cell differentiation but not in tangential migration (Garel et al., 1999). However, its role in $\mathrm{mDA}$ neuron development has not been investigated. Our data strongly suggest that Ebf1 is crucial for SNc formation during development.

Previous studies have shown that Ebfl is expressed in the striatum, in addition to the VM, during development, where it controls early cell differentiation (Garel et al., 1999; Lobo et al., 2006). However, several lines of evidence support our hypothesis that defective SNc formation results in striatal dysplasia in Ebf1deficient mice. First, loss of $E b f 1$ primarily results in defects in the striatal matrix where Ebf1 is exclusively expressed, whereas the patch compartment is well preserved until P0 (Garel et al., 1999). Thus striatonigral projections derived from the patch neurons are unlikely to be affected. Second, and importantly, matrix neurons are born at a late developmental stage, E15 or even E18-E21, and their innervations to the SNc would not be established until the early postnatal period (van der Kooy and Fishell, 1987; Hamasaki et al., 2001). Consistently, no significant apoptosis occurs in E15.5 homozygous mutant embryos. Ebf1 deficiency-induced apoptosis of matrix neurons is only observed after E18.5 (Garel et al., 1999). We also found no TUNEL ${ }^{+}$cells in the striatum in E15 Ebf1 null mutants (data not shown). Thus the timing for striatal dysplasia occurs much later than the deficiency in SN formation. Third, reelin has been reported to be produced in the striatum and transported to the SN (Nishikawa et al., 2003). We found that reelin expression was not altered in either the striatum or the SN in E15 Ebf1 ${ }^{-/-}$mutants (data not shown). Together, these data suggest that impaired SN formation is the primary cause of the striatal dysplasia.

\section{VM-enriched genes may be involved in distinct aspects of mDA neuron development}

Three VM-enriched genes, Fabp7, Ttyh1, and Hmgb2, may be novel molecules required for $\mathrm{mDA}$ neuron precursor activity as indicated by their very restricted expression in layers I and II. Fabp 7 may be involved in the maintenance of their self-renewal state, as its levels were constantly high from E10 to E14 (Fig. 3), and recent reports indicate a similar role in cortical neuroepithelial cells and radial precursor cells (Arai et al., 2005; Barry and McDermott, 2005). A role for Ttyh1 in cell adhesion and possibly as a transmembrane receptor in astrocytoma has also been suggested (Matthews et al., 2007). Similarly, Hmgb2, Id3, and cell cycle-related genes may play important roles in the proliferation of mDA neuron precursors but in a manner distinct from Fabp7. Their downregulation in the VM may be crucial for exit from the cell cycle. This is supported by previous findings that expression of nuclear Hmg2 (also known as Hmgb2) is one of the characteristics of undifferentiated cells (Angelova et al., 1993). These layer $\mathrm{I} / \mathrm{II}$-specific genes work together to retain $\mathrm{mDA}$ neuron precursor activities.

In the present study, many genes exhibit specific expression in layers III and/or IV, strongly suggesting that they play distinct roles in late VM development; Ebf1 is the first gene identified to be specifically responsible for the migration of nigral DA neurons. The fact that $E b f 1$ deficiency cannot be compensated for by the other Ebf gene family members $E b f 2$ or $E b f 3$, which are coexpressed in layer III, suggests that $E b f 2$ and $E b f 3$ may exert roles in distinct aspects of $\mathrm{mDA}$ neuron differentiation.

In conclusion, we delineated dynamic genetic maps of VM development from E10 to E14 and proposed a four-layer architecture of the VM. We identified a set of VM-enriched or layerspecific genes that regulate distinct aspects of $\mathrm{mDA}$ neuron development. Our data reveal molecular identities of developing mDA cells. Thus the present study lays the foundations for a better understanding of the molecular mechanisms underlying mDA development. 


\section{References}

Andersson E, Jensen JB, Parmar M, Guillemot F, Björklund A (2006) Development of the mesencephalic dopaminergic neuron system is compromised in the absence of neurogenin 2. Development 133:507-516.

Ang SL (2006) Transcriptional control of midbrain dopaminergic neuron development. Development 133:3499-3506.

Angelova A, Borissova Z, Avramova F, Simeonova V, Stambolova M (1993) HMG-2 protein in developing rat brain cells. Int J Biochem 25:37-41.

Arai Y, Funatsu N, Numayama-Tsuruta K, Nomura T, Nakamura S, Osumi N (2005) Role of Fabp7, a downstream gene of Pax6, in the maintenance of neuroepithelial cells during early embryonic development of the rat cortex. J Neurosci 25:9752-9761.

Barry D, McDermott K (2005) Differentiation of radial glia from radial precursor cells and transformation into astrocytes in the developing rat spinal cord. Glia 50:187-197.

Björklund A (2005) Cell therapy for Parkinson's disease: problems and prospects. Novartis Found Symp 265:174-186; discussion 187, 204-211.

Garcia-Dominguez M, Poquet C, Garel S, Charnay P (2003) Ebf gene function is required for coupling neuronal differentiation and cell cycle exit. Development 130:6013-6025.

Garel S, Marín F, Grosschedl R, Charnay P (1999) Ebfl controls early cell differentiation in the embryonic striatum. Development 126:5285-5294.

Garel S, Garcia-Dominguez M, Charnay P (2000) Control of the migratory pathway of facial branchiomotor neurones. Development 127: 5297-5307.

Hamasaki T, Goto S, Nishikawa S, Ushio Y (2001) A role of netrin-1 in the formation of the subcortical structure striatum: repulsive action on the migration of late-born striatal neurons. J Neurosci 21:4272-4280.

Kandel ER, Schwartz JH, Jessell TM (2000) Principles of neural science, Ed 4. New York: McGraw-Hill.

Kele J, Simplicio N, Ferri AL, Mira H, Guillemot F, Arenas E, Ang SL (2006) Neurogenin 2 is required for the development of ventral midbrain dopaminergic neurons. Development 133:495-505.

Lobo MK, Karsten SL, Gray M, Geschwind DH, Yang XW (2006) FACSarray profiling of striatal projection neuron subtypes in juvenile and adult mouse brains. Nat Neurosci 9:443-452.

Ma Q, Chen Z, del Barco Barrantes I, de la Pompa JL, Anderson DJ (1998) neurogenin 1 is essential for the determination of neuronal precursors for proximal cranial sensory ganglia. Neuron 20:469-482.

Matthews CA, Shaw JE, Hooper JA, Young IG, Crouch MF, Campbell HD
(2007) Expression and evolution of the mammalian brain gene Ttyh1. J Neurochem 100:693-707.

McKay R, Kittappa R (2008) Will stem cell biology generate new therapies for Parkinson's disease? Neuron 58:659-661.

Molyneaux BJ, Arlotta P, Menezes JR, Macklis JD (2007) Neuronal subtype specification in the cerebral cortex. Nat Rev Neurosci 8:427-437.

Nishikawa S, Goto S, Yamada K, Hamasaki T, Ushio Y (2003) Lack of Reelin causes malpositioning of nigral dopaminergic neurons: evidence from comparison of normal and Reln(rl) mutant mice. J Comp Neurol 461:166-173

Nunes I, Tovmasian LT, Silva RM, Burke RE, Goff SP (2003) Pitx3 is required for development of substantia nigra dopaminergic neurons. Proc Natl Acad Sci U S A 100:4245-4250.

Ohyama K, Kawano H, Asou H, Fukuda T, Oohira A, Uyemura K, Kawamura K (1998) Coordinate expression of L1 and 6B4 proteoglycan /phosphacan is correlated with the migration of mesencephalic dopaminergic neurons in mice. Brain Res Dev Brain Res 107:219-226.

Prakash N, Wurst W (2006) Genetic networks controlling the development of midbrain dopaminergic neurons. J Physiol 575:403-410.

Shults CW, Hashimoto R, Brady RM, Gage FH (1990) Dopaminergic cells align along radial glia in the developing mesencephalon of the rat. Neuroscience 38:427-436.

Simon HH, Saueressig H, Wurst W, Goulding MD, O’Leary DD (2001) Fate of midbrain dopaminergic neurons controlled by the engrailed genes. J Neurosci 21:3126-3134.

Smidt MP, Burbach JPH (2007) How to make a mesodiencephalic dopaminergic neuron. Nat Rev Neurosci 8:21-32.

Thuret S, Bhatt L, O'Leary DD, Simon HH (2004) Identification and developmental analysis of genes expressed by dopaminergic neurons of the substantia nigra pars compacta. Mol Cell Neurosci 25:394-405.

van der Kooy D, Fishell G (1987) Neuronal birthdate underlies the development of striatal compartments. Brain Res 401:155-161.

Wang SS, Lewcock JW, Feinstein P, Mombaerts P, Reed RR (2004) Genetic disruptions of $\mathrm{O} / \mathrm{E} 2$ and $\mathrm{O} / \mathrm{E} 3$ genes reveal involvement in olfactory receptor neuron projection. Development 131:1377-1388.

Yue Y, Widmer DAJ, Halladay AK, Cerretti DP, Wagner GC, Dreyer J-L, Zhou R (1999) Specification of distinct dopaminergic neural pathways: roles of the Eph family receptor EphB1 and ligand Ephrin-B2. J Neurosci 19:2090-2101. 